\title{
Dynamics of Coupled Contaminant and Microbial Transport in Heterogeneous Porous Media
}

\author{
Pacific Northwest National Laboratory \\ June 1,1997 \\ Progress Report
}

Principal Investigator

T.R. Ginn

(916) 752-1707 (Phone)

email: trginn@ucdavis.edu

172 Everson Hall

Department of Civil and Environmental Engineering

University of California, Davis

Davis, CA 95616-5294
Contributors and Collaborators

D.R. Boone

J.H. Cushman

\section{Co-Investigators}

M.M. Fletcher

(803) 777-3916 (Phone)

email: fletcher@biol.sc.edu

Belle W. Baruch Institute for Marine Biology and Coastal Research

University of South Carolina

Columbia, SC 29208

D.M. Friedrich

(509) 376-9637 (Phone)

email: dm_friedrich@pnl.gov

Environmental Molecular Sciences Laboratory

Pacific Northwest National Laboratory

P.O. Box 999, MSIN K8-96

Richland, WA 99352

E.M. Murphy

(509) 375-5914 (Phone)

email: em_murphy@pnl.gov

Environmental and Health Sciences Division

Pacific Northwest National Laboratory

P.O. Box 999, MSIN K3-61

Richland, WA 99352 


\section{Research Objective}

Dynamic microbial attachment/detachment occurs in subsurface systems in response to changing environmental conditions caused by contaminant movement and degradation.

Understanding the environmental conditions and mechanisms by which anaerobic bacteria partition between aqueous and solid phases is a critical requirement for designing and evaluating in situ bioremediation efforts. This interdisciplinary research project will provide fundamental information on the attachment/detachment dynamics of anaerobic bacteria in heterogeneous porous media under growth and growth-limiting conditions. Experiments will provide information on passive and active attachment/detachment mechanisms used by growing anaerobes capable of reductive dechlorination. Theoretical representations of these attachment/detachment mechanisms will be incorporated into existing groundwater flow and contaminant transport models that incorporate heterogeneity effects and can be used to predict behavior at field scales. These mechanistic-based models will be tested against experimental data provided through controlled laboratory experiments in heterogeneous porous media in large (meter-scale) 2-D flow cells. In addition to a mechanistic-based predictive model, this research will lead to new theories for the transient spatial distribution of microbial populations and contaminant plumes in heterogeneous porous media, improving our capability for designing staged remediation strategies for dealing with mixed contaminants.

\section{Research Statement}

The central challenge of in situ remediation strategies is the control of the transient spatial distribution of contaminants and remediation reagents (e.g., nutrients or microorganisms) in the presence of natural physical and chemical heterogeneities. Currently available predictive tools rely almost exclusively on the representation of passive attachment/detachment processes (e.g., filtration, sedimentation) with the bacterium treated as an inert biocolloid. One of the most intractable aspects of bioreactive transport, however, is active microbial partitioning (e.g., attachment/detachment) under growth and transport in physically and chemically heterogeneous systems. Active attachment/detachment is associated with microbial physiologic response to changes in local aqueous concentrations of nutrients and electron-acceptors: this process is treated rarely in field-scale bacterial transport theories, and not at all in available predictive tools.

Understanding coupled contaminant and bacterial transport in real subsurface media is critical to the success of potential future bioremediation/biobarrier strategies at DOE sites. Disposal of chlorinated hydrocarbons has generated extensive contaminant plumes in sand/gravel aquifers at DOE's Savannah River Site (SRS) in South Carolina and Paducah Gaseous Diffusion Plant (PGDP) in Kentucky, and such organic solutes interact with natural environments in coupled complex ways that have serious ramifications for remediation. For example, during the SRS in situ bioremediation demonstration, $90 \%$ of the increase in methanotroph biomass arising from degradation of trichloroethylene (TCE) partitioned into the aqueous phase. Initially, this methanotroph population was primarily attached to solid surfaces. If this partitioning behavior during bioremediation were understood and could be predicted, the knowledge could be exploited to effectively distribute the biomass across a contaminated area and to plan an injection 
network so as to minimize costly well construction and dampen or eliminate microbially induced reductions in hydraulic conductivity. This basic knowledge on the transport response of microorganisms is required for any subsurface remediation involving microbes, including staged approaches that sequentially degrade and immobilize multiple contaminants. Staged approaches (temporal or spatial) can exploit the different transport properties of distinct microbial populations and are especially well suited to DOE mixed contaminant problems.

The purpose of the proposed research is to address the limitations in understanding reactive contaminant transport and dynamic bacterial attachment/detachment under growth and growthlimiting conditions in natural porous media. This research will assess the coupled degradation and transport processes involving biological agents, with focus on the active and passive attachment/detachment and transport processes of anaerobic microorganisms involved in TCE degradation, including their persistence under transient variations in substrate and electronacceptor availability. Experiments will be performed in natural geologic materials that display general physical and chemical heterogeneity patterns representative of TCE-contaminated sandy media at SRS and at PGDP. The goals of the research are to develop basic knowledge of the fundamental relationship between contaminant transport and active bacterial transport and attachment/detachment under transient local chemical conditions. This knowledge will afford 'a quantitative understanding of the transient spatial distribution of contaminants and microbial populations during contaminant degradation and microbial growth in physically and chemically heterogeneous media. To accomplish this end, the proposed research includes an integrated effort toward the development of predictive models of contaminant transport and degradation in heterogeneous media, through a combined multi-scale experimental and theoretical approach.

\section{Research Progress}

The work and associated spending on this project are purposely accelerated during the second half of fiscal year 1997. The reason for this is threefold: 1) the DOE research contracts with key collaborators (Fletcher and Cushman) and the subcontract with Dr. Boone were not initiated until December 1996,2) the establishment of contracts and subcontracts delayed the search and hiring of post-doctoral associates, and 3) one of the principal investigators, Timothy Ginn, has moved to the University of California, Davis and the subcontracting to this PI has only recently been completed. This multidisciplinary research project is highly integrated. As such, the progress and results are also highly integrated among the different institutions. The collaborators met in October 1996 to discuss the selection of microbial strains and initial growth kinetics. An additional meeting is planned for July 1997 to enhance collaboration and interpretation of results

\section{Selection of Strains}

Four strains were selected for study in this project. Desulfomonile tiedjeii was selected because it is capable of dechlorination. The kinetics of this organism can be studied as a pure culture, both in suspension and in porous media, by providing it with a reduced substrate (e.g., formate) from which it can acquire the reducing equivalents it needs to dechlorinate. As well, this organism can be combined with another bacterial strain that can produce organic compounds 
such as formate. The propionate-oxidizing bacterium, strain LYP, that can oxidize propionate in co-culture with hydrogen- or formate-using strains, was selected. Thus, co-cultures of strain LYP and $D$. tiedjeii can be grown with propionate as the electron donor and one of several chlorinated compounds as electron acceptor. Two other bacteria that grow by using hydrogen and formate as electron donors were also selected. Co-cultures of strain LYP (which produces formate) with $D$. tiedjeii will be studied for kinetics of growth and interspecies formate transfer, but we can also add another formate-using bacterium as a third member to determine whether it can out-compete $D$. tiedjeii for formate. The two other formate-using bacteria selected are Desulfovibrio strain G-11 and Methanospirillum hungateii. These two species have very different thresholds of for-mate concentration that are required for formate uptake. In addition, the natural fluorescent properties of $M$. hungateii will be used to directly measure real-time, in situ biomass concentrations during the transport experiments (see In Situ Spectroscopy progress below). These methanogens contain the unique electron carrier, F420, that autofluoresces a blue green color with a peak at $420 \mathrm{~nm}$ when excited by UV light.

\section{Determination of Growth Kinetic Constants}

Experiments have begun at the Oregon Graduate Center to determine Monod constants for the growth of the four selected microbes. A 14-liter New Brunswick fermentor has been modified to serve as a continuous culture fermentor, with culture medium added by an highperformance liquid chromatograph (HPLC) pump and passing through a bacteriological filter inline to the fermentor. The first "shake-down" experiment in the fermentor is complete. In this experiment, Desulfovibrio strain G-11 with formate and sulfate were grown as substrates. The culture grew in the fermentor, demonstrating steady-state growth. After several modifications to the fermentor to enhance our ability to acquire samples, these experiments will be repeated in an attempt to determine the growth constants (Monod) in a dilute buffer solution that will be used for the adhesion experiments and kinetic studies of growth in saturated media. At the same time as the continuous culture studies, batch culture studies will be carried out to determine the maximum growth rate and the growth yield of this culture.

\section{Surface Characteristics of Anaerobes}

A post-doctoral associate was recently recruited to carry out the work on adhesion properties and kinetics of attachment/detachment of anaerobic microorganisms under the guidance of Dr. Fletcher at the University of South Carolina. Paula van Schie has just obtained her Ph.D. from Rutgers University, and was trained in anaerobic microbiology in Dr. Lily Young's laboratory. When she begins work at USC at the end of June, Dr. Schie will initially assess the adhesive properties of the test organisms and determine the influence of physiological conditions on degree and stability of adhesiveness. These experiments are detailed as follows.

Previous studies with aerobic organisms have demonstrated that cell adhesiveness can be affected by different nutrient conditions, such as types and concentration of available nutrients, carbon: nitrogen ratios, and degree of starvation of the organisms. The variability in adhesion with nutrient conditions varies with the organism; some show little change while others 
demonstrate significant changes in adhesiveness. Thus, adhesion properties of anaerobic organisms might be expected to alter with their physiological state. It is important to assess the adhesive properties of $D$. tiedjei and $M$. hungatii and the variability in these properties with growth and starvation conditions, because of their important roles in subsurface bioremediation. Adhesion is first assessed by batch kinetic assays, using flat coupons of glass and siliconized or iron-coated glass as test substrata. Test conditions include cells freshly grown in a nutrient-rich medium and cells starved in MSNS-PIPES buffer (a dilute buffer solution more representative of nutrient levels found in groundwater) for periods of 48 to 96 hours. After establishing the degree of variability in adhesiveness with the test strains, specific growth and maintenance conditions are established for batch kinetic adhesion experiments. Relative hydrophobicity and surface charge of the test organisms under different growth and maintenance conditions will also be determined by retention of cells on octyl- or phenyl-Sepharose beads and ion exchange resins. In this way the precise culture and maintenance conditions that must be used for adhesion assessment and kinetic experiments for these organisms can be assessed, so that these data are relevant to future transport experiments.

Preliminary measurements of surface charge and morphology have been made on the selected anaerobes in this project at Pacific Northwest National Laboratory (PNNL). The electrophoretic mobility of the different strains was measured using a Brookhaven Instruments-ZetaPlus analyzer. The charge of the particles is measured by applying an external electrical field and measuring the direction and rate of movement of the bacteria. The anaerobic strains were suspended in a dilute media that was designed to provide the necessary growth nutrients for the anaerobes, but at levels that are more realistic for groundwater. As expected for most bacteria, the membranes carried a net negative charge expressed as mobility (e.g., ratio of particle velocity in Fm s ${ }^{-1}$ to field strength expressed as Vcm-1). The mobilities measured were $-0.92,-2.72$, and -2.32 for M. hungateii, Desulfovibrio strain G-11, and D. tiedjei, respectively. These values are preliminary, but are similar to other reported values for bacteria (Bayer and Sloyer 1990). $M$. hungateii have flagella and are chemotactic, which presents an additional challenge for describing their transport in porous media.

The cell morphology affects transport in porous media and is also being measured for these selected strains. Preliminary analyses using a Macrotrac Ultrafine Particle Analyzer were inconsistent with cell size information found in the literature. This is likely because this analytical procedure is based on Stokes law for spherical particles. The anaerobic cells used in this project are not spherical. D. tiedjei is an elongated rod, 4-5 Fm long by -0.5 Fm wide, while $M$. hungateii cells are curved, 0.5 by $7 \mathrm{Fm}$ in diameter. Cell size is currently being assessed using phase-contrast microscopy.

\section{In Situ Spectroscopy}

As mentioned above, the natural fluorescent properties of methanogens, $M$. hungateii in particular, are being used to develop the capability to directly measure real-time, in situ biomass concentrations during the transport experiments. Fluorescence emission and excitation spectra of $M$. hungateii in dilute aqueous suspensions were measured at PNNL to determine optimum 
wavelengths for excitation and detection of the microbes in column and flow cell experiments. Original samples ( $\sim 5 \times 107$ cells $/ \mathrm{ml})$ were diluted 10 times to $\sim 6$ x 106 cells $/ \mathrm{ml}$ and placed in a $1 \mathrm{~cm} \times 1 \mathrm{~cm}$ fused quartz cuvette in the fluorometer. Excitation was by continuous xenon lamp through a double monochromator, which provided monochromatic light at selected wavelengths and protected the sample from scattered UV and visible light. Fluorescence was detected at right angles to the excitation direction through a double monochromator with a cooled, photoncounting photomultiplier detection system. Excitation spectra were measured by recording emission intensity near one of the fluorescence wavelength peaks $(426 \mathrm{~nm})$ as a function of the excitation wavelength, which was scanned. Emission spectra were measured by setting the excitation wavelength near one of the two excitation maxima (292 nm or 340-350 nm) followed by recording emission intensity as a function of the scanned wavelength of the emission monochromator.

Under UV excitation (290-350 nm) in the fluorometer, bright fluorescence was observed visually from the original, undiluted samples. The 10x diluted samples produced a fluorescence intensity of $-18,000$ counts/second. The intensity was approximately proportional to the concentration. Repeated scans indicate that the samples were relatively stable under continuous monochromatic UV radiation. Excitation maxima were observed at $292 \mathrm{~nm}$ (narrow band) and $340 \mathrm{~nm}$ (broad band) (Fig. la). The emission maxima depended on the excitation wavelength. Near UV excitation $(340 \mathrm{~nm})$ produced broad, somewhat structured blue emission peaking at $426 \mathrm{~nm}$ (Fig. 1b). Excitation in the quartz-UV (292 nm) produced a broad, unstructured near-UV emission band peaking around $365 \mathrm{~nm}$ (Fig. lc). The buffer solution showed no fluorescence in the absence of microbes. (Fig. lb).

These two excitation and emission band systems correspond to two different fluorophore centers in the microbes. The longer wavelength system (340 nm excitation, $426 \mathrm{~nm}$ emission) is suited for direct excitation and observation through pyrex (borosilicate) glass columns. Initial experiments will monitor transport of $M$. hungateii through 1" dia x 8" long glass columns filled with Accusand. Excitation will be from an argon ion laser $(351 \mathrm{~nm})$. Fluorescence will be detected through wavelength selective filters using a photon-counting photomultiplier.

\section{Modeling}

Significant advances have been made on two fronts in the modeling on this project at Purdue University. First, we have developed a multidimensional mathematical model representing sticky brownian motion with drift, as a general representation of microbial transport with attachment/detachment. This model has been implemented on the computer, and is being tested using data from Madilyn Fletcher's laboratory. A sticky brownian motion with drift allows a particle (e.g., microbe) to diffuse like a classical brownian motion in a pressure-induced convective field, except when it hits a wall. When it hits the wall, it sticks for a random period of time, which is dictated by a probabilistic stickiness condition. For comparison to Dr. Fletcher's data, the model is being applied within the experimental setting of the microflow 
chamber that is being used in her laboratory (i.e., the particles move in a similar dimensioned cell under similar pressure gradients). We are currently computing exit time probability densities as a function of peclet number and the flow cell length $(\mathrm{Pe}=\mathrm{d} / \mathrm{vl}$ where $\mathrm{v}$ is the flux, $\mathrm{d}$ is the diffusion coefficient, and 1 is the flow cell length).
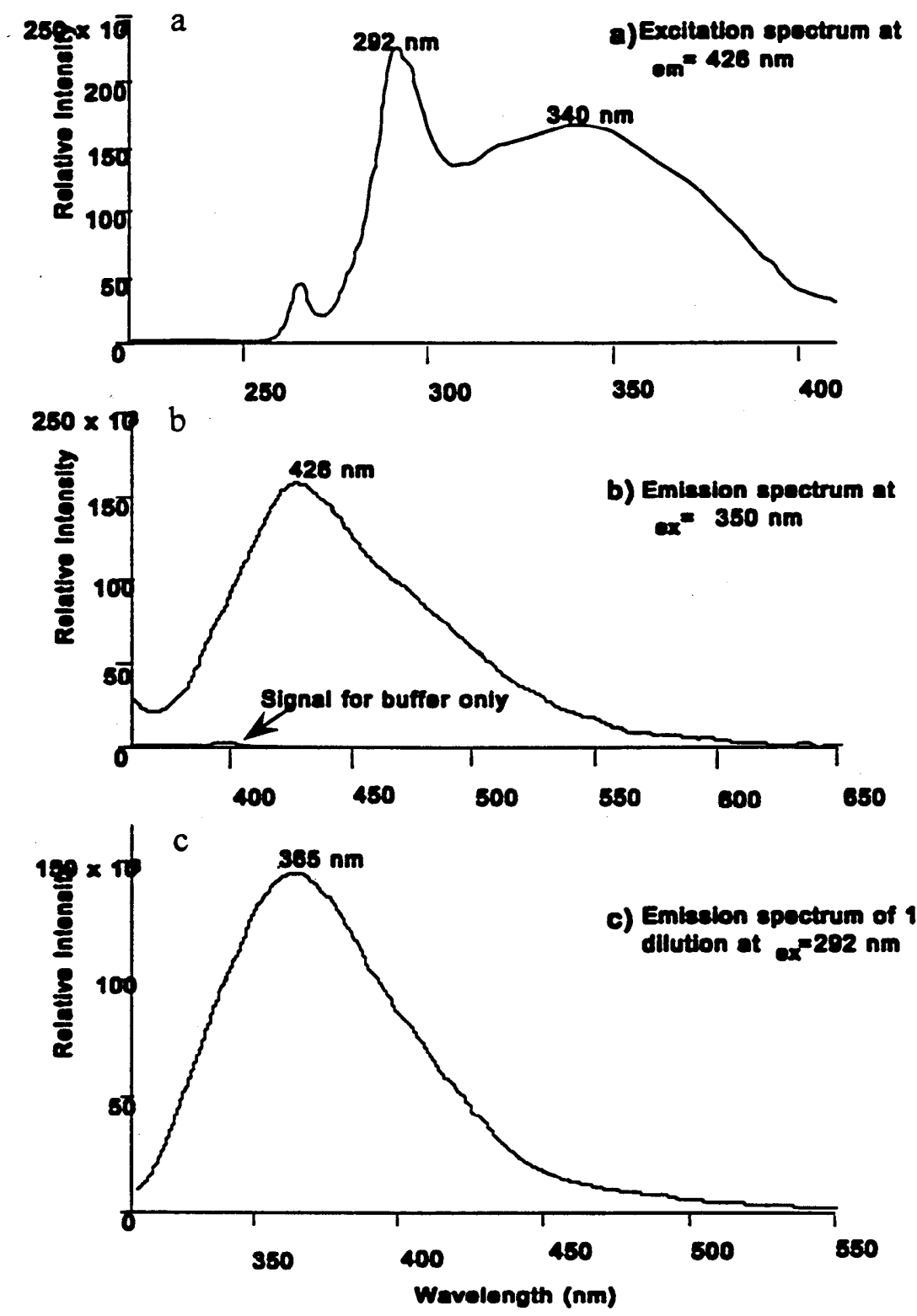

Figure 1. Excitation (a) and Emission (b) Spectra of M. Hungateii in Buffer Solution. The emission spectra (c) of the bacteria diluted $10 \%$ with excitation at $292 \mathrm{~nm}$. 
Second, a revolutionary result on upscaling stochastic transport of chemicals and bacteria has been obtained. Full characterization information of physical and chemical heterogeneity is rarely available for field sites, hence stochastic representations are often used to describe transport. Stochastic representations of transport arise in the presence of uncertain subsurface properties, which are treated as random (e.g., stochastic) variables. The resulting expressions for stochastic concentrations relate the statistical properties of concentration to the statistical properties of the porous media. These expressions involve an infinite series of statistical moments and thus have been impossible to solve explicitly (e.g., the expressions are not closed). This is known as the closure problem, and is inherent in upscaling any transport process in natural porous media. The closure problem has been solved and explicit upscaled solutions for the stochastic solute concentration, its moments, the macroscale dispersive flux, and the variance of fluctuating concentration for several standard problems have been produced. This solution is currently being implemented on the computer at the University of California, Davis so that actual transport problems can be tested. Because of the significance of this closure solution to the field of transport in porous media, a manuscript is in preparation and will be submitted this summer.

\section{Reference}

Bayer, M.E., and J.L. Sloyer, Jr., 1990, “The Electrophoretic Mobility of Gram-Negative and Gram-Positive Bacteria: An Electrokinetic Analysis,” J. Gen. Microbial. 136 :867-874. 